\title{
Assessment of the Treatment Response of Hepatocellular Carcinoma to Radiofrequency Ablation Using Dynamic and Diffusion MR Imaging according to LI-RADS v2018 Treatment Response Algorithm
}

\author{
SHAIMA F. ELKHOLY, M.D. and DALIA K. SEROUR, M.D. \\ The Department of Diagnostic and Interventional Radiology, Faculty of Medicine, Cairo University, Egypt
}

\begin{abstract}
Background: Hepatocellular carcinoma is the most common primary liver malignancy and considered as the fifth most common cancer worldwide. Resection and liver transplantation have a high survival in adequately selected patients, however locoregional ablative therapeutic techniques has several advantages over surgery like hepatic parenchymal preservation and overall have less morbidity and mortality.

Aim of Study: The aim of this study was to evaluate the role of diffusion and dynamic MR imaging in the assessment of the treatment response of hepatocellular carcinoma after radiofrequency ablation according to the LI-RADS treatment response v2018.
\end{abstract}

Patients and Methods: A retrospective study included 40 patients with $45 \mathrm{HCC}$ lesions underwent radiofrequency ablation and followed-up by dynamic and diffusion MR imaging. The data were collected from the PACS of Radiology Department, Cairo University Hospitals. The MR images were assessed according to LI-RADS 2018 treatment response algorithm.

Results: According to LI-RADS treatment response criteria, dynamic MRI has sensitivity of $100 \%$, specificity $95.8 \%$, Positive Predictive Value (PPV) $94.1 \%$ and Negative Predictive Value (NPV) $100 \%$ compared to sensitivity of $64.3 \%$, specificity $95.8 \%$, PPV $90 \%$ and NPV $82.1 \%$ for the diffusion weighted images in the detection of tumoural viability. Arterial Phase Hyperenhancement (APHE) was found in $87.5 \%$ of viable lesions while delayed wash out was found in $100 \%$ of the viable lesions.

Conclusion: Dynamic MRI is the cornerstone in the assessment of treatment response of hepatocellular carcinoma to radiofrequency ablation. Arterial phase hyperenhancement and delayed wash out are the main criteria for diagnosis of tumoural viability according to LI-RADS treatment response algorithm. Diffusion imaging and ADC measurement are good negative techniques for exclusion of tumoural activity and also can confirm the dynamic findings in viable cases.

Correspondence to: Dr. Dalia K. Serour, E-Mail: daliakhaled80@yahoo.com
Key Words: HCC - RFA - Diffusion MRI - LI-RADS - Treatment response.

\section{Introduction}

HEPATOCELLULAR Carcinoma (HCC) is a major health problem and one of the commonest causes of cancer related death worldwide. It is considered as the most common primary malignancy of the liver and the fifth most common malignancy in the world among men and the seventh among women $[1,2]$. A rapid rising rate of $\mathrm{HCC}$ among Egyptian patients is noted in the last few years with almost two-fold increase. Liver cirrhosis secondary to chronic viral hepatitis infection is the major risk factor for development of HCC [3]

Several therapeutic options are available for management of HCC depending on several factors including the size and site of the lesion, the stage of the tumor at patient's presentation as well as the general condition of the patient and eligibility for surgical intervention $[4,5]$. Unfortunately, patients meet the transplantation criteria and are candidate for surgical resection only in 5-10\% of cases [6]

\footnotetext{
Abbreviation:

APHE : Arterial Phase Hyperenhancement.

AUC : Area Under Curve.

DWI : Diffusion Weighted Images.

EASL : European Association for the Study of the Liver. HCC : Hepatocellular Carcinoma.

LI-RADS : Liver Imaging Reporting and Data System.

NPV : Negative Predictive Value.

PACS : Picture Archiving and Communication System.

PPV : Positive Predictive Value.

RECIST : Response Evaluation Criteria in Solid Tumors.

RFA : Radiofrequency Ablation.

ROC : Receiver Operating Characteristic.

ROI : Region of Interest.

TACE : Transcatheter Arterial Chemoembolization.
} 
Locoregional therapies have been emerged as a curative treatment option in well selected patients and also it can serve as a bridge for liver transplantation by down-staging of the tumour to meet transplantation criteria. Thermal ablation (radiofrequency and microwave ablation) and Transcatheter Arterial Chemoembolization (TACE) are the most widely performed locoregional procedures [7]. Radiofrequency ablation showed comparable results to surgical resection as regards the survival rate particularly, in patients with small HCC lesions $(<2 \mathrm{~cm})$ who are not potential candidates for liver transplantation [8].

Proper and accurate evaluation of the treatment response after radiofrequency ablation therapy is crucial for further therapeutic planning, as early identification of tumoural residue or recurrence after treatment is critical to decide whether a repeated treatment cycle or another treatment option are needed before disease progression [5,9].

The choice of imaging modality to be used for assessment based on the availability, institutional preference and reader's experience, with $\mathrm{CT}$ is more widely used than MRI [5]. However, MRI has several advantages over CT such as more obvious contrast resolution, image subtraction and the functional techniques like diffusion and perfusion imaging that provide the ability to detect microscopic changes in the tumor cellularity and vascularity. Familiarity with normal and abnormal imaging appearance after RFA is crucial for accurate assessment of the treatment response [10,11].

On diffusion weighted images, the ablation zone usually appears of hypointense signal at its center while the margins of the lesion may show hyperintensity at high $b$ value with low ADC due to hyperemia and cytotoxic edema. So, it is a challenge for the radiologist to differentiae normal post-treatment parenchymal changes from active tumors [12].

WHO criteria and the Response Evaluation Criteria in Solid Tumors (RECIST) were used for assessment of treatment response depending on the change in the tumor's size. However such criteria doesn't reflect the actual changes in the tumour vascularity and tissue necrosis. Moreover; larger size of the ablation zone compared to the HCC lesion before treatment is considered a normal finding early after ablation as ablation [10] .

EASL (European Association for the Study of the Liver) criteria and mRECIST have been emerged due to the previously mentioned limita- tions. Such criteria take into account the cytostatic response of the lesion (i.e. the change in the size of the enhancing component rather than the change in tumor size) [10]

The LI-RADS (Liver Imaging Reporting and Data Systems) response algorithm was introduced at 2017 and modified at v.2018. It based mainly upon assessment of arterially enhancing tumor similar to mRECIST, but it expands upon the mRECIST as it includes atypical enhancing tumors, introduces the "non evaluable" category in case of degraded image quality and also includes the treatment expected imaging findings after different types of ablation. LI-RADS also facilitates the communication between the radiologist and the treating physician using a common language $[10,13]$

In this study, our aim was to evaluate the role of MRI using dynamic and diffusion imaging techniques in the assessment of the treatment response of HCC after RFA using LI-RADS v.2018 treatment response algorithm and to determine whether diffusion imaging can differentiate between the malignant lesions and the benign post ablation parenchymal changes.

\section{Patients and Methods}

This retrospective study included 40 patients with $45 \mathrm{HCC}$ lesions underwent radiofrequency ablation procedure. The data were collected from the PACS of our institution including the period between January 2015 and December 2017.

Written informed consent was signed by all patients before the MRI examination.

All the patients had liver cirrhosis on top of chronic viral hepatitis.

The dynamic MRI examination was done for all patients in the early post ablation period (within the first 4-6 weeks). Another follow-up MRI study was preformed after 3-4 months in patients showing no definite radiological signs of disease recurrence or residue.

\section{Inclusion criteria:}

- Patients with HCC lesions underwent only RFA as interventional procedure.

- Patients with no absolute MRI contraindications.

Exclusion criteria:

-Absolute MRI contraindications or contraindications to contrast media.

- Patients having liver tumors other than HCC. 
- Patients underwent therapeutic procedures other than RFA.

- Patients with combined RFA and systemic therapy.

\section{MRI technique:}

- The MRI technique was done using Philips 1.5 Tesla MRI scanner (Achieva-Netherlands). Precontrast images were first obtained including axial $\mathrm{T} 1$, axial T2, axial SPAIR and coronal $\mathrm{T} 1$ and $\mathrm{T} 2$ images. For $\mathrm{T} 1 \mathrm{WIs}$ the acquisition parameters were (TR $250 \mathrm{~ms}$, TE $30 \mathrm{~ms}$, Flip angle $15^{\circ}$, FOV 300-350, slice thickness: 7) and for T2 WIs were (TR $1000 \mathrm{~ms}$, TE $80 \mathrm{~ms}$, flip angle $90^{\circ}$, FOV 300-350, Slice thickness: 7).

- The diffusion imaging were acquired using respiratory triggered technique (single-shot spin echo echoplanar sequence) with the following acquisition parameters (TR $1700 \mathrm{~ms}$, TE $76 \mathrm{~ms}$, slice thickness 8mm, b-values 0, 500, 1000 $\mathrm{mm}^{2} / \mathrm{s}$ ).

- Dynamic study was then performed after manual injection of $0.1 \mathrm{mmol} / \mathrm{kg}$ body weight Gd-DTPA followed by $20 \mathrm{ml}$ saline flushing. Imaging was done using 3D T1-weighted fat-suppressed gradient sequence (THRIVE). The dynamic study consisted of a pre contrast series followed by four post contrast series (early arterial, late arterial "arterio-portal", portal and venous phases) with 19-21 seconds intervals. This includes 17 seconds for image acquisition and 3-4 seconds for rebreathing. A delayed phase after 5 minutes was then obtained. The imaging was done at end expiration to limit the misregistration artifact. The acquisition parameters were (TR $4.4 \mathrm{~ms}$, TE $2.2 \mathrm{~ms}$, flip angle $10^{\circ}$, FOV $300-350$, slice thickness: $2-3 \mathrm{~mm}$ ).

\section{Interpretation of the MR images:}

- Post processing of the acquired MR images was done by two experienced abdominal imaging radiologists reading independently using the Phillips workstation (extended MR workspace 2.6.3.5 Netherlands 2011).

- The analysis include the morphological features of the lesions (number, size, margins and signal at $\mathrm{T} 1$ and $\mathrm{T} 2$ weighted images), the enhancement pattern of the ablation zone and any suspicious lesions at different phases of the study and then analysis of the diffusion images.

Interpretation of the dynamic study:

-We followed the LI-RADS TR algorithm in assessment of the treatment response.
- Arterial Phase Hyperenhancement (APHE) is defined as hyperenhancement of the entire lesion or part of the lesion relative to background liver parenchyma during the arterial phase [14]

- The APHE is categorized as rim or non rim enhancement.

- Rim enhancement is the type of arterial enhancement which is most pronounced in the observation periphery [14] and usually seen in other malignancies and categorized as LR-M [15]. While non rim enhancement is one of the major features of HCC [14].

- The APHE requires both hyperenhancement and hyperintensity relative to the liver during the arterial phase. This means that, if a hypointense lesion showed contrast uptake but still not brighter than the liver in the arterial phase, APHE is not present [14]. The arterial phase subtraction images were then interpreted to confirm or to exclude the presence of enhancement by removing the precontrast high $\mathrm{T} 1$ signal form the images, so the residual hyperintensity is due to enhancement.

- Delayed wash out is defined as visually assessed temporal reduction in the enhancement, within the whole or a part of the lesion, relative to the background liver [14]

- The delayed wash out is morphologically characterized as nonperipheral or peripheral wash out. Non-peripheral washout is a major imaging features for HCC while peripheral washout is feature of LR-M [15]

- Delayed enhancing capsule is considered as major feature of HCC.

Interpretation of the diffusion weighted images:

- Qualitative analysis: Areas of high signal at diffusion images should be correlated with ADC map to exclude $\mathrm{T} 2$ shine through effect.

- ADC measurement: ADC maps were automatically generated on the workstation using the three $b$ values. A circular region of interest (ROI) was drawn including the area to be measured.

- The ADC values of the whole ablation zones, surrounding parenchymal changes and areas suspicious for recurrent/residual lesions were calculated for the different study groups.

- The diffusion observation was categorized into:

- Definite diffusion restriction: If the area of interest appeared of high signal in the diffusion images (higher than the background parenchyma) and low signal at the ADC map. 
- Facilitated diffusion: If the area of interest appeared of low signal (similar or lower than the parenchyma) in the diffusion images and high signal at the ADC map.

- Uncertain: If the area of interest showed faint sustained hyperintensity in the diffusion images and no corresponding hypointensity on ADC maps. Considering the tiebreaking rules of LIRADS TR algorithm which state that, if the reader was unsure between two categories, choose the category reflecting lower certainty, the uncertainty in the diffusion analysis was considered as negative for malignancy.

Interpretation of enhancement surrounding the ablation zone:

- An ill defined early enhancement of the hepatic parenchyma surrounding the treatment zone that usually persists in the delayed phase is considered as post-treatment effect "treatment specific enhancement pattern" and caused by reactive tissue hyperemia, inflammation and post treatment perfusion alteration (vascular injury or arterioportal shunts).

-Well defined delayed rim enhancement of the ablation zone is also considered as treatment specific enhancement pattern and caused by granulation tissue formation.

- Well defined nodular, mass like or thick irregular tissue enhancement along the margins or within the treatment zone which shows APHE or delayed wash out are considered as viable tumoural tissue. The size of viable tumor (whether equivocally, probably, or definitely viable) was measured according to LI-RADS recommendation as the longest dimension of the enhancing area, not traversing non enhancing area. The measurement could be done at the arterial phase or the areas of wash out at the delayed phase (unlike mRECIST criteria in which the tumour was measured at the enhancing phase only).

The patients were then categorized into the following groups according to LI-RADS TR algorithm:

- LR-TR non evaluable: If the images could not be properly evaluated due to image degradation.

- LR-TR nonviable "probably or definitely non viable": If there's no lesion enhancement or there's treatment specific enhancement. Complete disappearance of the lesion is equivalent to absence of enhancement and categorized as non viable.
- LR-TR equivocal "equivocally viable": If the enhancement pattern is atypical for treatment specific enhancement and also don't meet the criteria for tumoural viability.

- LR-TR viable "probably or definitely viable": If there's nodular, mass like or thick irregular tissue enhancement along the margins or within the treatment zone which shows APHE or delayed wash out or enhancement pattern similar to the pre intervention pattern. No threshold growth criterion for LR-TR viable.

- Tiebreaking rules were applied: If the reader was unsure between two categories, we choose the category reflecting lower certainty i.e. if lower certainty of non viability choose LR-TR equivocal and if lower certainty of viability also choose LR-TR equivocal.

- Ancillary features defined by LI-RADS TR criteria including T2 hyperintensity and diffusion restriction. Features may help in identification of suspected active tumors on dynamic images. However, they are still not included in the Treatment Response Algorithm [10]

\section{Reference standard and follow-up:}

- Pathological study is the gold standard in confirmation of tumour activity, however we didn't depend on pathology as most of the patients do not undergo surgery and don't obtain a tissue biopsy as it's technically difficult for small lesions and may result in sampling error.

- The reference standard was the features of the lesion in the dynamic MRI study according to LI-RADS treatment response algorithm. Followup is needed in patients categorized as non viable and equivocal.

\section{Statistical analysis:}

Unpaired student $t$-test was used to compare the mean ADC values of the different study groups and the results are expressed as mean \pm standard deviation or number (\%). $p$-value $\leq 0.05$ was considered as significant and $<0.01$ was considered as highly significant.

\section{Results}

40 patients with $45 \mathrm{HCC}$ lesions were included in this study. Two patients with two HCC lesions were excluded from the statistical analysis due to image degradation and categorized as LR-TR non evaluable and assessed with CT instead of dynamic MRI. Other 3 patients with $3 \mathrm{HCC}$ lesions were also excluded due to missed follow-up studies on our PACS. The final cohort is 35 patients ( 8 females 
and 27 males) with 40 HCC lesions. Their ages ranged between 51 and 71 years and the mean age was 60.8 years.

According to the LI-RADS TR algorithm, we found that 24 lesions (60\%) were categorized as LR-TR non viable and 16 lesions (40\%) were LRTR viable.

The signal intensity of the recurrent lesions were assessed in the precontrast T1 and T2 weighted images and it was found that 13 out of the 16 malignant lesions (about 81.2\%) exhibit high T2 signal and only 6 out of the 16 lesions $(37.5 \%)$ exhibit low $\mathrm{T} 1$ signal.

Regarding the types of recurrence in the LRTR viable group: 10 lesions (62.5\%) showed nodular type recurrence, 4 lesions $(25 \%)$ showed mass like recurrence and 2 lesions (12.5\%) appeared as thick irregular tissue Fig. (1). The HCC lesion before treatment may show atypical enhancement pattern like rim enhancement and the recurrence may show similar atypical pattern, however we didn't meet such cases.

Regarding the pattern of enhancement of the malignant lesion in the dynamic study:

- 14 malignant lesions out of 16 lesions (87.5\%) show APHE. 5 lesions (35.7\%) in the early arterial phase (1 ${ }^{\text {st }}$ phase in the dynamic study which obtained about 10-25 seconds after contrast injection) and 9 lesions (64.3\%) in the late arterial (arterio-portal phase) which obtained about 2540 seconds after contrast injection. The enhancement is proved by the subtracted images.

- Wash out was found in all the 16 malignant lesions.

Regarding the treatment specific expected enhancement in the dynamic study:

- An ill defined early enhancement of the hepatic parenchyma surrounding the treatment zone that persists or become isointense to the liver in the delayed phase Fig. (2) was found in 29 lesions including all the 24 LR-TR non viable lesions and 5 of the 16 malignant lesions in the LR-TR viable group imaged at the early post ablation period (4-5 weeks after ablation). The benign parenchymal enhancement detected in the non viable group was resolved at the 6 months followup in 9 cases $(37.5 \%)$ and at 12 months followup in 13 cases $(54.1 \%)$ while we didn't find further follow-up for the remaining two cases.

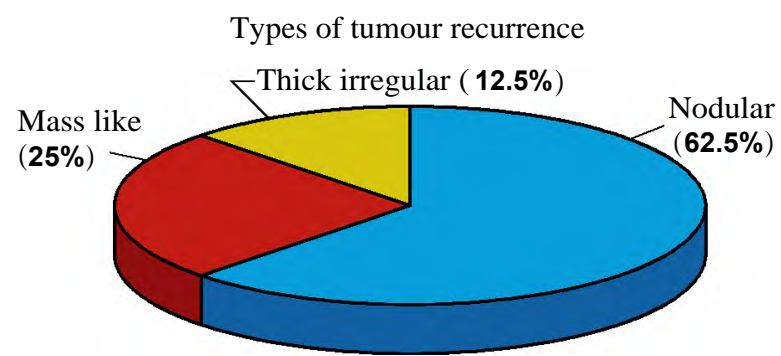

Fig. (1): Pie chart demonstrating the types of tumoural recurrence in the LR-TR viable group.
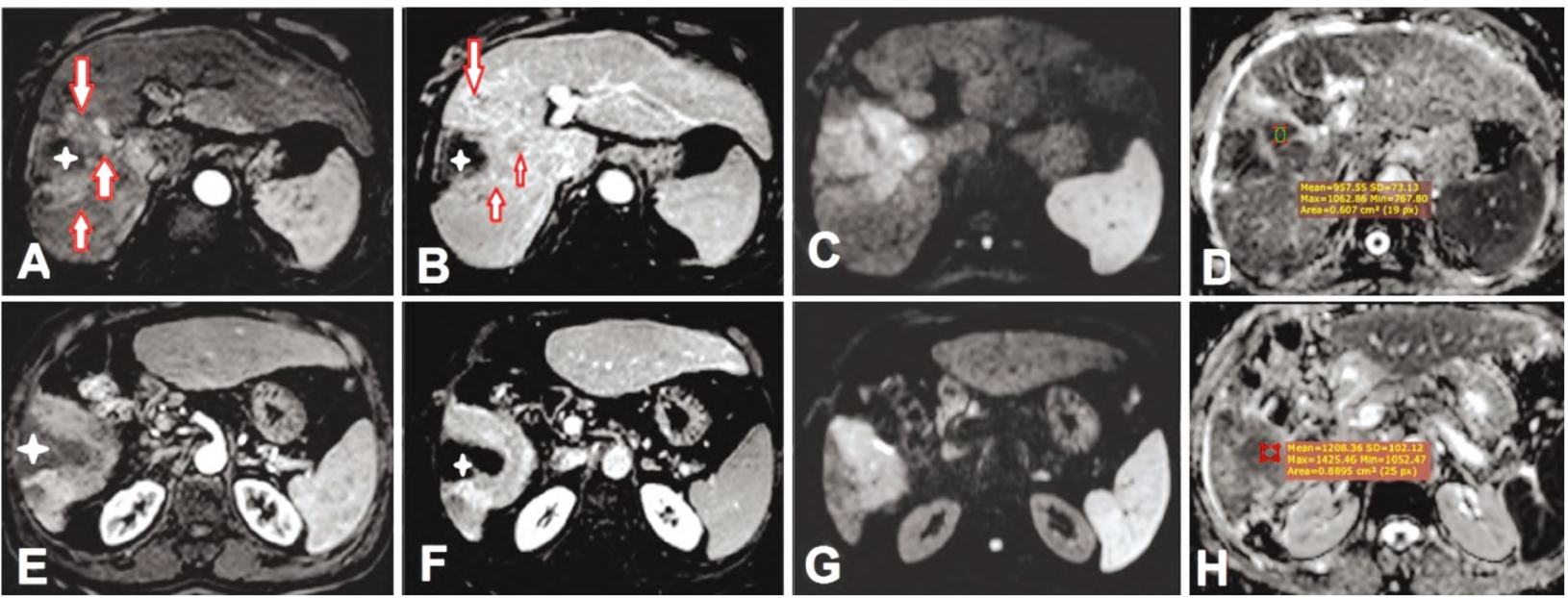

Fig. (2): 54 year old male patient underwent RFA for a right hepatic lobe $\mathrm{HCC}$ and imaged 1 months after the procedure. (A) Axial arterial phase of the dynamic study showing multiple thick irregular enhancing lesions along the margins of the treatment zone. (B) Delayed phase of the study showing wash out of the enhancing lesions denoting tumoural activity. (C, D) Diffusion weighted images and ADC map showed the marked diffusion restriction and low ADC of the enhancing areas $(0.95$ $\mathrm{X} 10^{-3}$ ). (E) Axial arterial phase of the dynamic study at a lower level showing patchy parenchymal areas of faint arterial enhancement surrounding the treatment zone (asterisk) (F) The enhancement became more evident and homogenous in the delayed phase suggesting benign post treatment hyperemia and perfusions alteration. $(\mathrm{G}, \mathrm{H})$ Diffusion weighted images and ADC map showed the mild increased signal in the diffusion images and higher ADC value of the enhancing areas $\left(1.2 \times 10^{-3}\right)$. 
- Delayed rim enhancement of the ablation zone (granulation tissue formation) was found in 17 of the lesions in the LR-TR non viable group imaged at the 6 th month after ablation procedure and persists in all cases in the next follow-up.

\section{Diffusion results and ADC values:}

The ADC values of the 16 viable lesions ranged between 0.86 and $1.27 \times 10^{-3} \mathrm{~mm}^{2} / \mathrm{s}$ (mean $1.02 \pm$ $0.127)$. The ADC values of benign parenchymal post-treatment changes found in 24 lesions ranged from 1.11 to $1.43 \times 10^{-3} \mathrm{~mm}^{2} / \mathrm{s}$ (mean $1.31 \pm 0.101$ ). A statistical significant difference was found $(p$ 0.0001).

Table (1) shows the correlation between the dynamic MRI findings and the reference standard. The lack of APHE represents good therapeutic response in $95.9 \%$ of LR-TR non-viable cases (i.e.

Table (1): Correlation between the dynamic findings and the reference standard.

\begin{tabular}{lccccc}
\hline \multirow{2}{*}{$\begin{array}{l}\text { Dynamic } \\
\text { results }\end{array}$} & \multicolumn{2}{c}{ LR-TR viable } & & \multicolumn{2}{c}{ LR-TR nonviable } \\
\cline { 2 - 3 } & Number & $\%$ & & Number & $\%$ \\
\hline TP & 16 & 100 & & \\
FN & 0 & 0 & & \\
FP & - & - & & 4.1 \\
TN & - & - & & 23 & 95.9 \\
\hline Total & 16 & 100.0 & & 24 & 100.0 \\
\hline
\end{tabular}

true negative), while APHE and/or delayed washout represent tumoral viability in $100 \%$ of LR-TR viable cases. Thus the dynamic MRI has sensitivity of $100 \%$, specificity $95.8 \%$, PPV $94.1 \%$ and NPV $100 \%$.

Table (2) shows the correlation between the diffusion findings and the reference standard. The lack of diffusion restriction represents good therapeutic response in $95.9 \%$ of LR-TR non-viable cases (i.e. true negative), while diffusion restriction represent tumoral viability only in $68.8 \%$ of LR cases (true positive) and the remaining cases (31.2\%) in the LR-TR viable group showed facilitated diffusion (false negative) Figs. $(3,4)$. Thus the diffusion images has sensitivity of $64.3 \%$, specificity $95.8 \%$, PPV $90 \%$ and NPV $82.1 \%$. Table (3) illustrates the difference in the statistical indices of the diffusion and dynamic findings.

Table (2): Correlation between the diffusion findings and the reference standard.

\begin{tabular}{lccccc}
\hline \multirow{2}{*}{$\begin{array}{l}\text { Diffusion } \\
\text { results }\end{array}$} & \multicolumn{2}{c}{ LR-TR viable } & & \multicolumn{2}{c}{ LR-TR nonviable } \\
\cline { 2 - 3 } TP & Number & $\%$ & & Number & $\%$ \\
FN & 5 & 68.8 & & \\
FP & - & 31.2 & & 1 & 4.1 \\
TN & - & - & & 23 & 95.9 \\
\hline Total & 16 & 100.0 & 24 & 100.0 \\
\hline
\end{tabular}
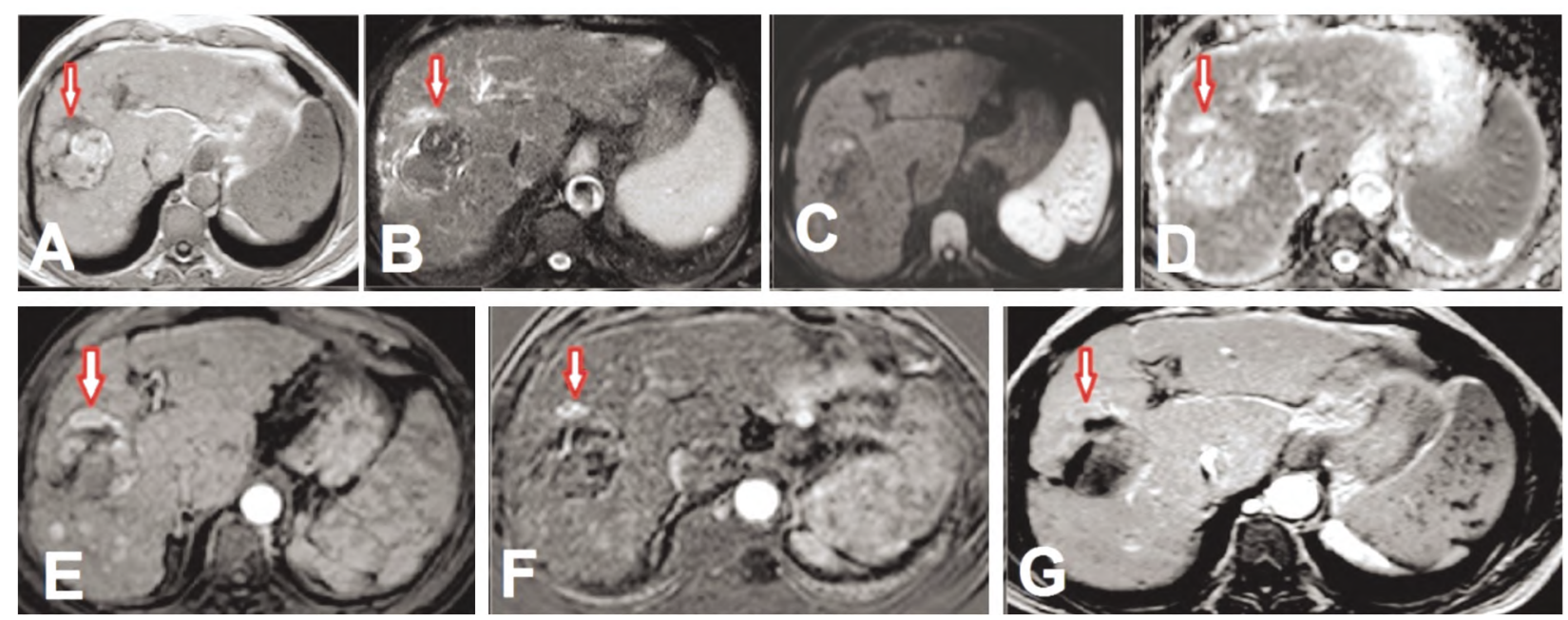

Fig. (3): 60 year old male patient underwent RFA for a right hepatic lobe HCC and imaged 3 months after the procedure. (A) Axial T1 WI, (B) Axial T2 SPAIR WI showing the treatment zone at the right lobe exhibiting high T1 and low T2 signal with a small nodule at the anterior margins of the treatment zone exhibiting low T1 and high T2 signal "arrowed". (C) Diffusion images and (D) ADC map showed facilitated diffusion of the suspicious nodule. (E) Arterial phase of the dynamic study and (F) Delayed phase of the dynamic study showing APHE and delayed wash out of the nodule in keeping with tumoural activity (false negative result for diffusion images). 

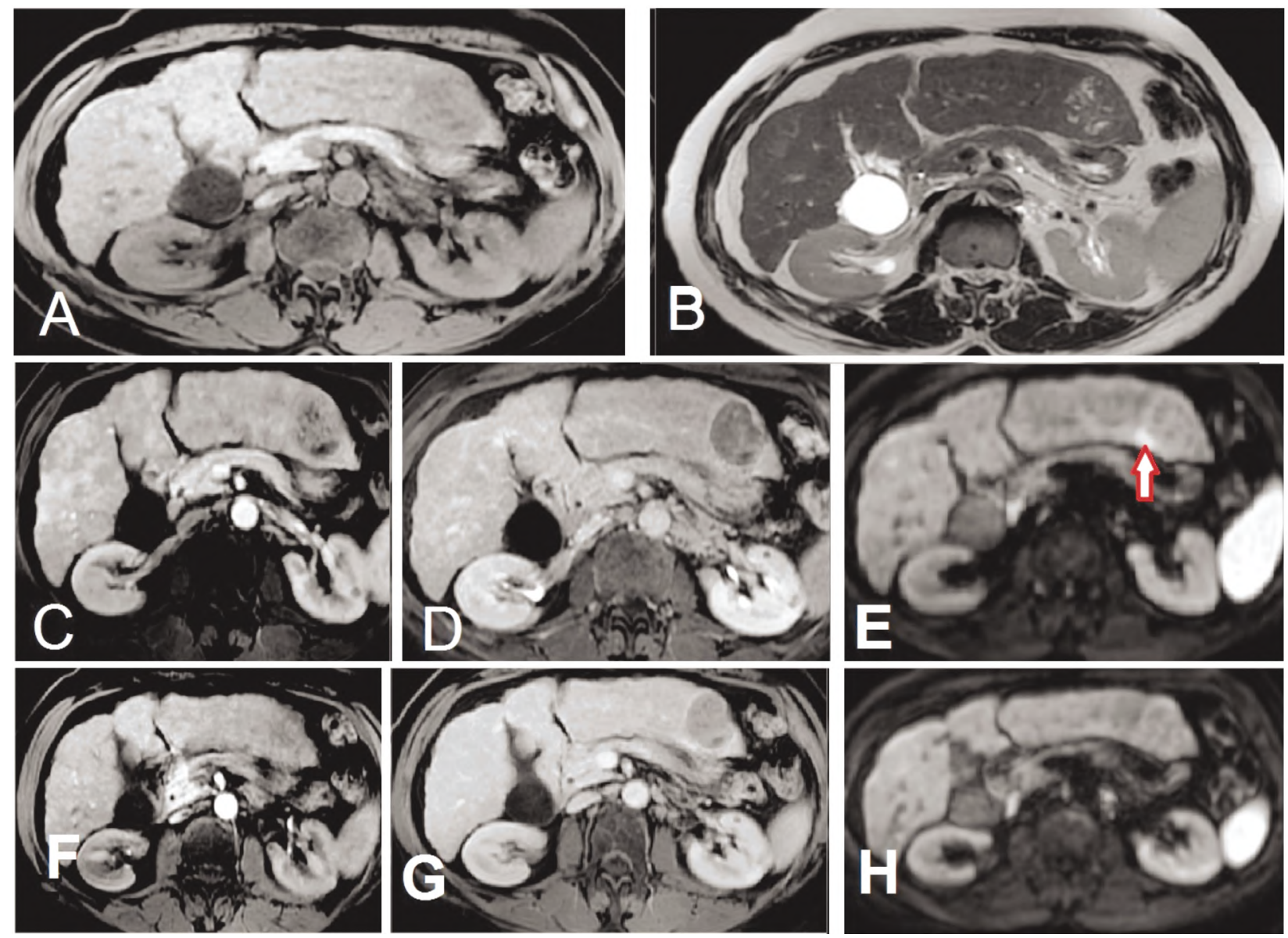

Fig. (4): 53 year old female patient underwent RFA for a left hepatic lobe HCC and imaged 6 months after the procedure. (A) Axial T1 WI, (B) Axial T2 SPAIR WI showing the treatment zone at the left lobe exhibiting low T1 and intermediate T2 signal with areas of liquefaction within. (C) Arterial phase of the dynamic study and (D) Delayed phase of the dynamic study showing no evidence of suspicions APHE or delayed wash out along the margins of the treatment zone. (E) Diffusion images showed a small nodule with restricted diffusion at the posterior margins of the treatment zone (false positive result for diffusion images). (F) Arterial phase of the dynamic study and (G) Delayed phase of the dynamic study performed 3 months later and showing regression in the size of the treatment zone with still no evidence of suspicions APHE or delayed wash out $(\mathrm{H})$ Diffusion images showed disappearance of the suspicious nodule.

Table (3): Difference in the statistical indices of the dynamic MRI and the diffusion imaging.

\begin{tabular}{lcc}
\hline Statistics & Dynamic results Diffusion results \\
\hline Sensitivity & $100 \%$ & $64.3 \%$ \\
Specificity & $95.8 \%$ & $95.8 \%$ \\
Positive predictive value & $94.1 \%$ & $90 \%$ \\
Negative predictive value & $100 \%$ & $82.1 \%$ \\
\hline
\end{tabular}

\section{Receiver Operating Characteristic (ROC) curve:}

ROC curves are commonly used to characterize the sensitivity/specificity tradeoffs for a binary classifier. The area under the curve is viewed as a measure of a forecast's accuracy that donated by AUC. Table (4) and Fig. (5) are showing that the AUC of diffusion category and ADC measurements for the different study groups was with confidence interval range between 0.674 and 0.972 producing a significant $p$-value equals 0.001 .

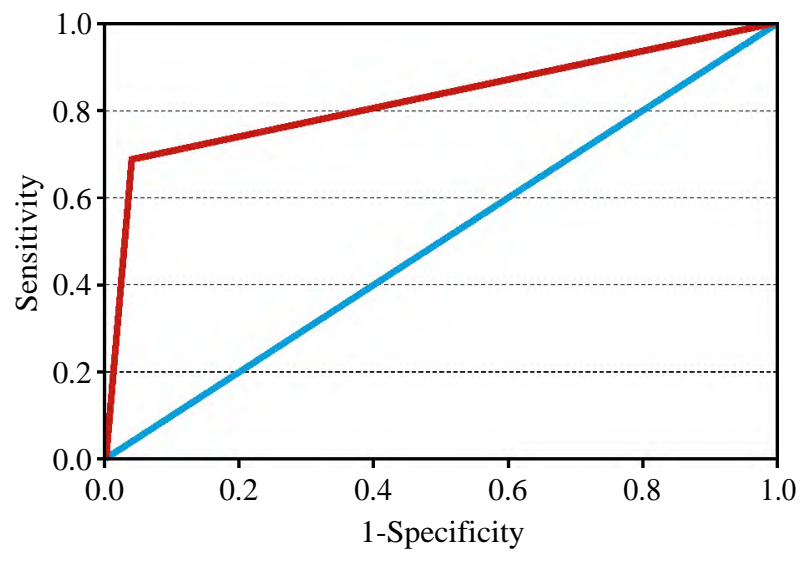

Fig. (5): ROC curve for diffusion weighted images. 
Table (4): ROC curve analysis for diffusion and ADC analysis showing the AUC and 95\% confidence interval.

\begin{tabular}{|c|c|c|c|c|c|c|}
\hline \multirow{2}{*}{ Test results } & \multirow{2}{*}{$\begin{array}{l}\text { Standard } \\
\text { error }\end{array}$} & \multirow{2}{*}{$\begin{array}{c}p- \\
\text { value }\end{array}$} & \multicolumn{2}{|c|}{$95 \%$ confidence interval } & \multirow{2}{*}{ Sensitivity } & \multirow{2}{*}{ Specificity } \\
\hline & & & Lower bound & Upper bound & & \\
\hline Diffusion category 0.823 & 0.076 & 0.001 & 0.674 & 0.972 & $68.8 \%$ & $95.8 \%$ \\
\hline
\end{tabular}

\section{Discussion}

HCC is the fifth most common cancer worldwide and the most common primary liver malignancy [17]. RFA is a widely accepted and widely used thermal ablative technique inducing cell death via alternating electrical current to destroy malignant cells [13]. Proper assessment of the treatment response and early detection of recurrent or residual malignant tissue is crucial for patient's management and indicates the need for further therapeutic options as early as possible before disease progression. LI-RADS TR algorithm is the most recently published criteria for assessment and categorization of the HCC lesion after ablation and standardize the imaging definitions used and improve consistency in reporting and guide further patient management.

The aim of this study was to evaluate the role of MRI using dynamic and diffusion imaging techniques in the assessment of treatment response of HCC after RFA using LI-RADS v.2018 treatment response algorithm and to determine whether diffusion imaging can differentiate between the malignant lesions and the benign post ablation parenchymal changes.

The identification of normal findings after ablation is essential and radiologists should be familiar with such findings in order to detect the signs of tumoural activity. The presence of few air bubbles early in the first few days after ablation can be considered as a normal post ablation finding, however the diagnosis of infection and abscess formation should be considered if the gas bubbles are persisting or increasing. The size of the ablation zone is larger than the lesion before treatment as ablation should includes safety margins. While size reduction usually begins about 6 months after ablation [10].

Assessment of the treatment response according to LI-RADS TR algorithm can increase the reader's confidence and standardize the terms used in reporting, however large studies assessing the treatment response using LI-RADS criteria with pathological correlation are lacking.
A recent study done by Cools et al., on forty five patients with 81 tumors (59 ablated and 22 untreated) and correlated their results with histopathology after liver transplantation found that $39 \%$ of the ablated lesions had viable HCC on histopathology. The sensitivity/specificity of LR-TR categories of ablated tumors was $30 \% / 99 \%$, with a PPV/NPV of 93\%/69\%. The Inter-Rater Reliability (IRR) was high (90\% agreement. They concluded that, the LR-TR criteria after ablation has high IRR but low sensitivity. The low sensitivity could be explained by the disruption in the local blood flow after thermal ablation which could affect the arterial enhancement on MRI [18] .

Our results showed that $\mathrm{T} 2$ hyperintensity which is an ancillary feature in LI-RADS TR algorithm is superior to non enhanced T1 hypointensity in detection of tumour recurrence. Such finding could be explained by the high contrast between low signal of the ablation zone and high signal of the malignant lesion. However early after ablation, the heterogenous $\mathrm{T} 2$ signal of the treatment zone could mask tumour recurrence. Our findings agreed with Mahmoud et al., who performed a study on 50 patients with HCC underwent RFA or MWA and they found that $77 \%$ of the recurrent lesions elicit high T2 signal and only $31 \%$ of the lesions elicit low T1 signal [9]. This also agreed with Dromain et al., who found all the recurrent lesions exhibiting hyperintense T2 signal without suspicious areas being detectable on T1-weighted images [19].

According to the LI-RADS TR criteria, the pattern of tumour recurrence may be nodular, mass like or thick irregular tissue. We found that 10 lesions $(62.5 \%)$ showed nodular type recurrence, 4 lesions $(25 \%)$ showed mass like recurrence and 2 lesions (12.5\%) appeared as thick irregular tissue. This agreed with Dromain et al., who detected $77.5 \%$ of the recurrent lesions as small nodules at the ablation zone margins and $22.5 \%$ of the lesions as thick irregular rim [19]. Also this agreed with Mahmoud et al. who found the nodular type as the most common recurrence and detected in $76.9 \%$ of cases [9]. In another study performed on 50 patients with HCC underwent RFA, It was found 
that nodular recurrence was detected in $84.6 \%$ of patients [20].

Delayed wash out was found to be the most specific sign for tumoural activity, as in our study it was found in $100 \%$ of malignant lesions while APHE was found in $87.5 \%$ (35.7\% of which was found in the early arterial phase and $64.3 \%$ in the late arterial phase). This indicates that dynamic study with multiple arterial and portal phases should be performed rather than the old technique using fixed time delay to ensure that at least one of the arterial phases will detect the tumoural enhancement. Also dynamic MRI is more superior to triphasic $\mathrm{CT}$ in detection of malignant lesions owing to its ability to obtain multiple dynamic phases in short time without exposure to ionizing radiation.

An ill defined enhancement of the hepatic parenchyma surrounding the treatment zone that persists or become isointense to the liver in the delayed phase is considered as treatment specific expected enhancement and could be explained by the reactive tissue edema and perfusional changes and usually seen in most cases early after ablation and resolve gradually. Several previous studies had reported similar findings. The largest study was done by Kierans et al., who performed a study over 203 ablated lesions and they found that the perilesional enhancement seen in $53.7 \%$ of cases imaged $<4$ months, that persists in $25.6 \%$ at $4-9$ months and only in $12.2 \%$ in cases imaged $>9$ months after RFA [21]. Also Mahmoud et al., who found that such finding was present in $100 \%$ of patients imaged within the 1 st month after ablation and persists in $9 \%$ of patients imaged at 4-6 months and only in 5\% after 9-12 months [9]. Schraml et al., also reported perilesional ill-defined area of diffusion hyperintensity in $22.5 \%$ of the patients imaged within the first 6 months after the ablation. They found it to persist in $19.5 \%$ in the next followup study at 6-9 months and only in $6.5 \%$ of patients at 9-12 months [22].

Delayed rim enhancement of the ablation zone (granulation tissue formation) was also considered as a benign treatment related expected enhancement. Such findings was proved to be benign and detected by several previous studies $[\mathbf{9 , 1 9 , 2 2 ]}$. It should be assessed carefully as recurrence of tumoural activity is mostly found at the periphery of the ablation zone explained by the fact that maximum temperature during ablation is achieved within the center of the lesion while the periphery of the treatment zone showed the lowest temperature during ablation, hence the recurrence is commonly occurred at the margins of the treatment zone. However the recurrence is mostly nodular or thick irregular tissue and showed arterial enhancement and/or delayed wash out in opposition to the granulation tissue which is thin and uniform and showed persistent delayed enhancement.

Diffusion MR imaging can differentiate between the post-treatment benign parenchymal changes and the malignant lesions based on the fact that therapeutically induced changes including inflammation, hemorrhage, edema and necrosis have lower cellularity than tumoural tissue and therefore present lower signal in DWI and higher ADC values. The mean $\mathrm{ADC}_{3}$ values of the viable lesions was $1.02 \pm 0.127 \times 10^{-3} \mathrm{~mm}^{2} / \mathrm{s}$ while that of benign parenchymal post-treatment changes was $1.31 \pm$ $0.101 \times 10 \mathrm{~mm} / \mathrm{s}$. A statistical significant difference was found ( $p$ 0.0001). Our findings showed that diffusion imaging can provide a good negative results in detection of malignancy. Our results agreed with Schraml et al., who performed the largest reported study over 54 patients after RFA and analyzed the 148 follow-up MR examinations. They found that the mean ADC value of the recurrent lesions differs significantly from the mean $\mathrm{ADC}$ value of the surrounding parenchymal tissue changes ( $p$-value 0.0124) [22]. Also agreed Mahmoud et al., who found that the mean ADC value of the post-treatment reactive tissue changes (1.29 $\pm 0.12 \times 10^{-3} \mathrm{~mm}^{2} / \mathrm{s}$ ) which was significantly higher than the mean ADC of the recurrent or residual malignant lesions $\left(0.91 \pm 0.09 \times 10^{-3} \mathrm{~mm}^{2} / \mathrm{s}\right)$ with $p$-value 0.0001 [9]. In another study performed over 50 HCC lesions underwent RFA, a significant statistical difference between the benign tissue changes and the malignant lesions was found with a cut off value $1.05 \times 10^{-3} \mathrm{~mm} / \mathrm{s}$ [20].

\section{Limitations:}

The study design is a retrospective study which has some limitations inherited to this study design. The comparison between the pre and post-treatment MRI is important for proper assessment of the treatment response especially in cases with atypical enhancement pattern before treatment as the recurrence in such cases usually present similar atypical enhancement pattern. In this retrospective study, the pre-treatment MRI was not found in all cases which is considered one of the limitations of our study, however we depends on the other criteria for assessment of the treatment response. It's advised that to use the Treatment Response Algorithm with caution in cases with combined locoregional and systemic therapy as the effects of systemic treatment can impact the post-treatment appearances in unpredictable ways [10]. The term LR-TR non viable means that there is no gross viable 
tumor, while small foci of viable tumour cells could not be totally ruled out by MRI or other non invasive imaging modalities; so that, the non viability defined by LI-RADS TR algorithm is an imaging definition used to improve consistency in reporting and guide further patient management [13], further studies with large number of patients is recommended with correlation between imaging and pathological findings.

\section{Conclusion:}

Dynamic MRI is the cornerstone in the assessment of treatment response of hepatocellular carcinoma to radiofrequency ablation. Arterial phase hyperenhancement and delayed wash out are the main criteria for diagnosis of tumoural viability according to LI-RADS treatment response algorithm. Diffusion imaging and ADC measurement are good negative techniques for exclusion of tumoural activity and can also confirm the dynamic findings in viable cases.

\section{References}

1- THOMAS A.J., MENIAS C.O., PICKHARDT P.J., et al.: Bleeding Liver Masses: Imaging Features With Pathologic Correlation and Impact on Management. A.J.R., 213: 816. doi.org/10.2214/AJR.19.21240, 2019.

2- MITTAL S. and EL-SERAG H.B.: Epidemiology of hepatocellular carcinoma: Consider the population. J. Clin. Gastroenterol., 47 (suppl): S2-S6, 2013.

3- SHAKER M.K.: Epidemiology of HCC in Egypt. Gastroenterol Hepatol., 4 (3): 00097, 2016.

4- LIN S., HOFFMANN K. and SCHEMMER P.: Treatment of hepatocellular carcinoma: A systematic review. Liver Cancer, 1: 144-58, 2012.

5- HUSSEIN R.S., TANTAWY W. and ABBAS Y.A.: Insights into Imaging, 10: 8 https://doi.org/10.1186/s13244-0190690-1, 2019.

6- ELSAID N.A., KADDAH R.O., FATTAH M.S. and SALAMA N.M.: Subtraction MRI versus diffusion weighted imaging: Which is more accurate in assessment of hepatocellular carcinoma after Trans Arterial Chemoembolization (TACE)? The Egyptian Journal of Radiology and Nuclear Medicine, 47 (4): 1251-64, 2016.

7- KULIK L.M. and CHOKECHANACHAISAKUL A.: Evaluation and management of hepatocellular carcinoma. Clinics in liver disease, 19 (1): 23-43, 2015.

8- CHINNARATHA M.A., CHUANG M.Y., FRASER R.J., WOODMAN R.J. and WIGG A.J.: Percutaneous thermal ablation for primary hepatocellular carcinoma: A systematic review and meta-analysis. J. Gastroenterol. Hepatol., 31 (2): 294-301, 2016.

9- MAHMOUD B.E., ELKHOLY S.F., NABEEL M.M., et al.: Role of MRI in the assessment of treatment response after radiofrequency and microwave ablation therapy for hepatocellular carcinoma. The Egyptian Journal of Radiology and Nuclear Medicine, 47 (2): 377-85, 2016.

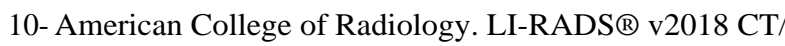
MRI Manual. https://www.acr.org/Clinical-Resources/ Reporting-and-Data-Systems/LI-RAD S/CT-MRI-LIRADS-v2018. accessed July 2018.

11- CHOI J.Y., LEE J.M. and SIRLIN C.B.: CT and MR imaging diagnosis and staging of hepatocellular carcinoma: Part I. Development, growth and spread: Key pathologic and imaging aspects. Radiology, 272: 635-54, 2014.

12- HOFFMANN R., REMPP H., SCHRAML C., et al.: Diffusion-weighted imaging during MR-guided radiofrequency ablation of hepatic malignancies: Analysis of immediate pre-and post-ablative diffusion characteristics. Acta Radiol., 56 (8): 908-16, 2015.

13- KIELAR A., FOWLER K.J., LEWIS S., et al.: Locoregional therapies for hepatocellular carcinoma and the new LI-RADS treatment response algorithm. Abdom. Radiol. (NY), 43: 218-30, 2018.

14-ELSAYES K., FOWLER K., CHERNYAK V., et al.: User and System Pitfalls in Liver Imaging With LI-RADS. J. Magn. Reson. Imaging. DOI: 10. 1002/jmri.2683 9, 2019.

15- FOWLER K.J., POTRETZKE T.A., HOPE T.A., et al.: SR. LI-RADS M (LR-M): Definite or probable malignancy, not specific for hepatocellular carcinoma. Abdom. Radiol., 43: 149-57, 2018.

16- CHOI J.Y., LEE J.M. and SIRLIN C.B.: CT and MR imaging diagnosis and staging of hepatocellular carcinoma. Part 2. Extracellular agents, hepatobiliary agents, and ancillary imaging features. Radiology, 273: 30-50, 2014.

17- WILLATT J., RUMA J.A., AZAR S.F., DASIKA N.L. and SYED F.: Imaging of hepatocellular carcinoma and image guided therapies-how we do it. Cancer Imaging, 17 (1), 9, 2017.

18- COOLS K.S., MOON A., BURKE L., et al.: Validation of the LI-RADS Treatment Response criteria after thermal ablation for hepatocellular carcinoma. American Association for the Study of Liver Diseases. doi: 10.1002/ 1t.25673, 2019.

19- DROMAIN C., De BAERE T., ELIAS D., et al.: Hepatic tumors treated with percutaneous radio-frequency ablation: CT and MR imaging follow-up. Radiology, 223: 255-62, 2002.

20- MOSTAFA M.M.S.: Diffusion weighted and dynamic contrast enhanced magnetic resonance imaging in assessment of malignant liver tumors after percutaneous radiofrequency ablation. The Egyptian Journal of Radiology and Nuclear Medicine, 47: 1195-205, 2016.

21- KIERANS S., ELAZZAZI M., BRAGA L., et al.: Thermoablative Treatments for Malignant Liver Lesions: 10Year Experience of MRI Appearances of Treatment Response. A.J.R., 194: 523-9, 2010.

22- SCHRAML C., SCHWENZER N., CLASEN S., et al.: Navigator Respiratory-Triggered Diffusion-Weighted Imaging in the Follow-up after Hepatic Radiofrequency Ablation-Initial Results. Journal Of Magnetic Resonance Imaging, 29: 1308-16, 2009. 


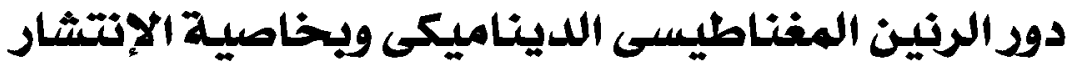

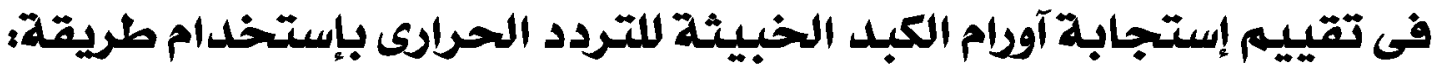

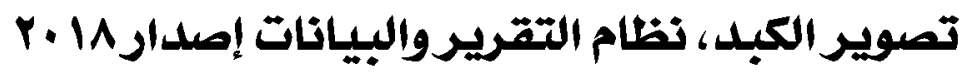

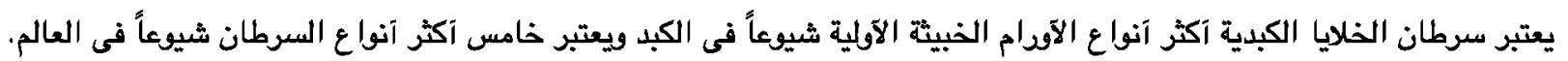

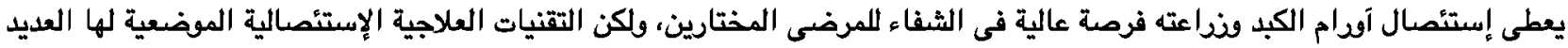

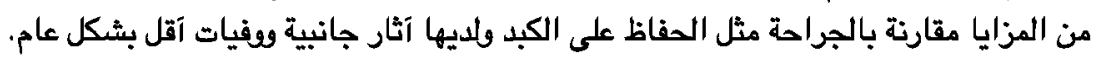

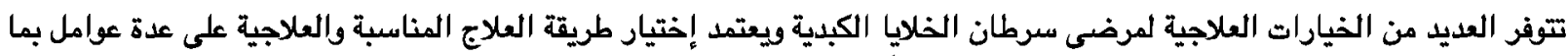

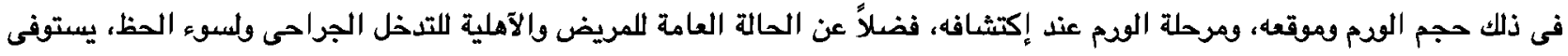

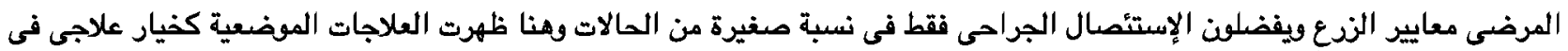

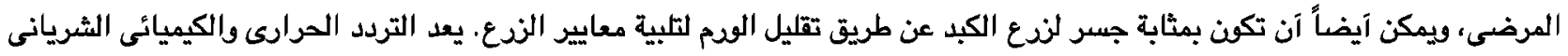

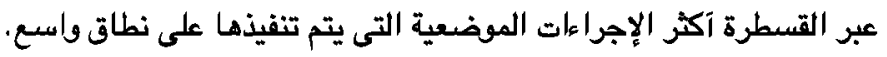

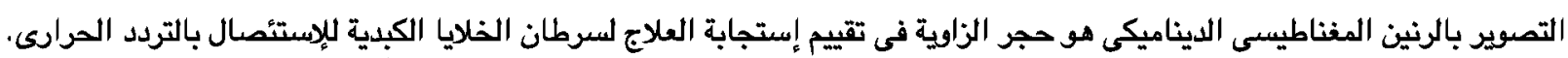

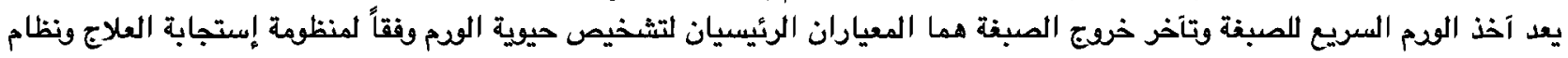
اليانات.

يعد التصوير بالإنتشار والقياس الظاهر للإنتشار من التقنيات الجيدة لإستبعاد نشاط الودم ويمكنه آيضاً تاكيد النتائج الديناميكية فى 